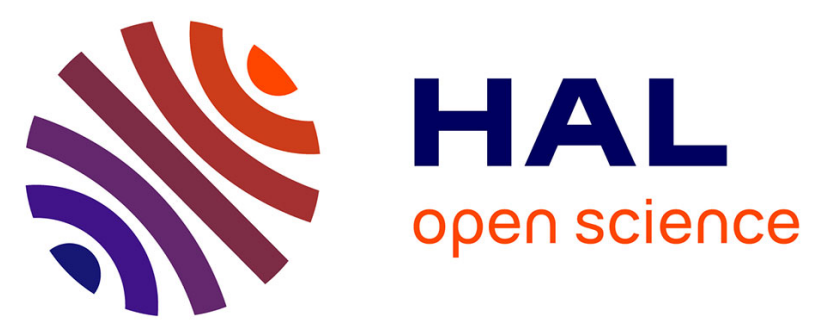

\title{
Fast Evaluation of the Fatigue Lifetime of Elastomers Based on a Heat Build-up Protocol and Micro-tomography Measurements
}

\author{
V. Le Saux, Yann Marco, Sylvain Calloch, Cédric Doudard, P. Charrier
}

\section{To cite this version:}

V. Le Saux, Yann Marco, Sylvain Calloch, Cédric Doudard, P. Charrier. Fast Evaluation of the Fatigue Lifetime of Elastomers Based on a Heat Build-up Protocol and Micro-tomography Measurements. International Journal of Fatigue, 2010, 32 (10), pp.1582-1590. 10.1016/j.ijfatigue.2010.02.014 hal00493227

\section{HAL Id: hal-00493227 \\ https://hal.science/hal-00493227}

Submitted on 23 Aug 2011

HAL is a multi-disciplinary open access archive for the deposit and dissemination of scientific research documents, whether they are published or not. The documents may come from teaching and research institutions in France or abroad, or from public or private research centers.
L'archive ouverte pluridisciplinaire HAL, est destinée au dépôt et à la diffusion de documents scientifiques de niveau recherche, publiés ou non, émanant des établissements d'enseignement et de recherche français ou étrangers, des laboratoires publics ou privés. 


\title{
Fast evaluation of the fatigue lifetime of rubber-like materials based on a heat build-up protocol and micro-tomography measurements
}

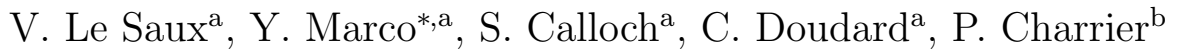 \\ ${ }^{a}$ Laboratoire Brestois de Mécanique et des Systèmes (EA 4325), \\ ENSIETA/Université de Brest/ENIB, 2 rue F. Verny 29806 Brest Cedex 9, France \\ ${ }^{b}$ Trelleborg Modyn, Z.I. Nantes Carquefou BP 419, 44474 Carquefou Cedex, France
}

\begin{abstract}
The temperature of rubber-like materials increases under cyclic loadings, due to their dissipative behaviour and low thermal conductivity. This well-known phenomenon, called heat build-up, has attracted the attention of researchers for a long time. But, to our knowledge, no published studies have tried to link this temperature rise to fatigue life behaviour, as already done for many metallic materials. Two main points are discussed in this paper. The first one is dedicated to heat-build up measurements: a specific experimental protocol was developed to capture the instantaneous heat build-up and, based on this protocol, a "heat build-up test" was defined in order to link the temperature rise to the principal maximum strain, which is a commonly used variable for fatigue life criterion. A discussion on the correlation between these results and the fatigue life behaviour is opened. This relation is illustrated for several industrial materials by a comparison between heat build-up measurements and fatigue life duration. The second point investigates the ability to couple X-ray tomography measurements presented elsewhere [1] to the former heat build-up results in order to predic the initiation lifetime. An approach based on a critical energy criterion was proposed and the comparison to a classic Wöhler curve approach gave very good results.
\end{abstract}

Key words: rubber-like materials, heat build-up, infrared thermography, $\mathrm{X}$-ray micro-tomography, critical energy criterion

\footnotetext{
*Corresponding author. Tel.: 33 (0)2 983489 11; Fax: 33 (0)2 98348730

Email address: Yann.Marco@ensieta.fr (Y. Marco)
} 


\section{Introduction}

Rubber-like materials are extensively used in industrial applications because of their ability to undergo large deformations and of their damping behaviour. Elastomeric components used in the automotive industry, such as engine mounts or torque rod, are submitted to cyclic loadings and a good conception towards fatigue phenomenon is therefore mandatory to ensure the safety of these structures. Fatigue initiation properties are studied by submitting specimens of a given geometry to a given cyclic load and measuring the number of cycles needed to reach an end-of-life criterion (presence of a crack of a given length, fracture of the specimen or stiffness loss). From these results, the so-called Wöhler curve, or "S/N" curve (Stress or Strain vs Number of cycles) is built. To be reliable, this classical method presents at least two main disadvantages: it requires long duration tests and a large number of specimens (usually a minimum of 25 specimens is required) in order to have a good estimation of the fatigue intrinsic dispersion. These two disadvantages obviously limit the study of fatigue life properties and the determination of the influences of some parameters (i.e. mean load, amplitude load, etc.) on these properties. To reduce this cost (of time and money), other methods have to be developed.

For several years, different methods for the rapid estimation of mean fatigue limits of metallic materials, based on temperature measurements, have been developed [2] and references therein. The aim of this paper is to investigate the opportunity to use these methods for rubber-like materials. In a first part, a heat build-up experiment and the associated analysis suitable for metallic materials is reminded. Based on this protocol, a heat build-up experiment suitable for rubber-like materials is proposed in a second part. Taking into account the specific aspects of this kind of materials, the experiment links the temperature rise to the maximum principal strain. In a third part, the relevance of a link between thermal measurements and fatigue life properties is discussed from the results measured on fifteen industrial materials. Even if this empirical approach gave some interesting results, it was also observed that, as expected, the viscosity has a first order influence on the temperature rise. It was therefore difficult to identify the dissipated energy only related to the fatigue mechanisms. These fatigue mechanisms are strongly linked to the flaw population and to the cavities created along a 
fatigue test. In a forth part we consequently use the microstructural information obtained from a X-ray computed micro-tomography investigation [1] to evaluate the ratio of the global dissipated energy to the one related to the fatigue damage. A critical energy criterion is applied and the results are compared to a Wöhler curve obtained classically. This comparison exhibits a very good correlation, opening a very promising field of investigation.

\section{Material and testing}

\subsection{Material and specimens}

A wide range of elastomeric materials, based on industrial standard recipes are used in this study. The aim was to cover a wide range of materials, crystallizing, non crystallizing, unfilled, monomer or copolymers matrix with a wide range of mechanical properties (fatigue lifetime, $\tan \delta$ ). More than 15 materials were tested. Hourglass shaped specimens were manufactured from a single batch in order to ensure the reliability of mixing and moulding conditions. The geometry of the specimen is given on figure 1. This kind of specimen (called AE2 in the following) was chosen for two main reasons: it is classically used to obtain Wöhler curves and the initiation and break zone is well controlled and is located in the thinner section.

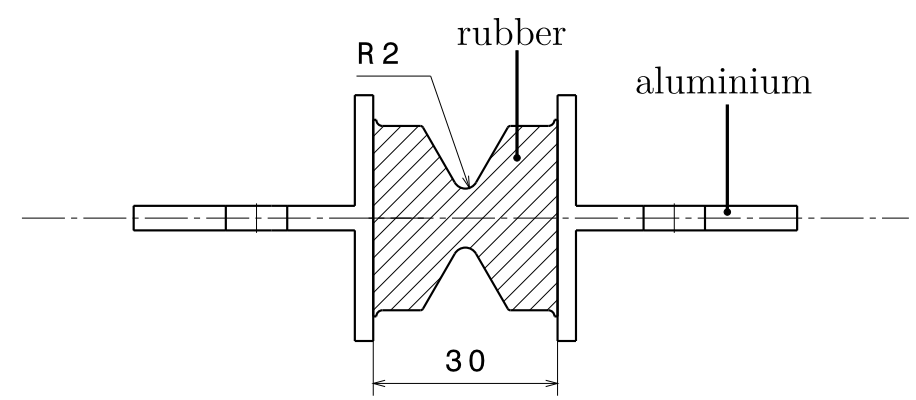

Figure 1: AE2 specimen

\subsection{Heat build-up based protocol for metallic materials}

For several years, different methods for the rapid estimation of mean fatigue limits of metallic materials based on temperature measurements have been developed [2]. They consist in applying successive sets of a given number of cycles for different increasing stress levels (figure 2(a)). For each stress level, the change of the temperature difference $\theta=T-\frac{T_{1}+T_{2}}{2}$ (where $T$ is 

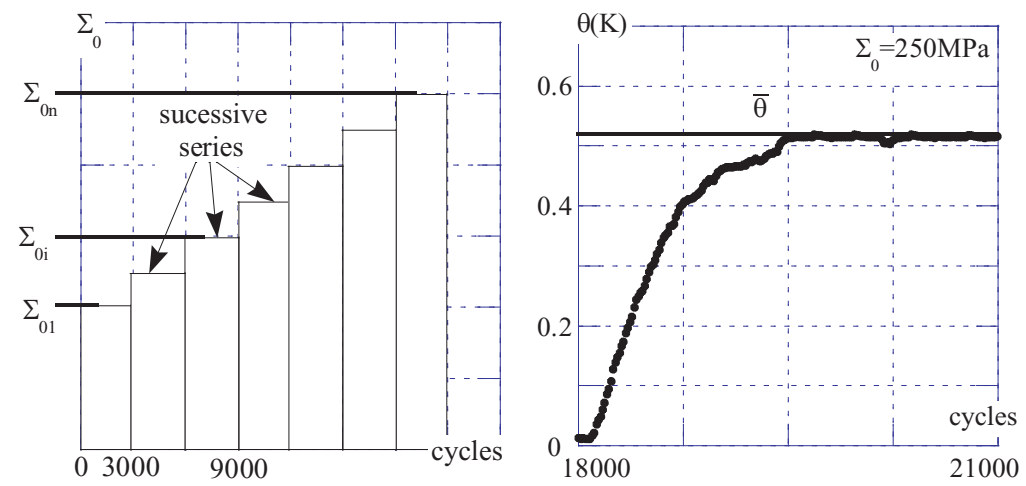

(a) Successive series for various amplitude stress levels

(b) Evolution of the mean temperature during a loading serie

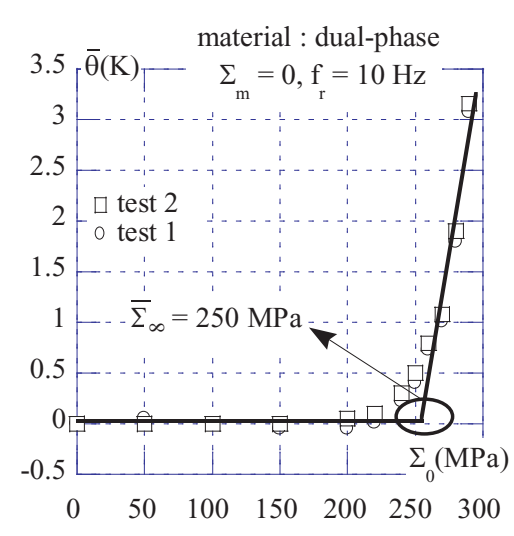

(c) Evolution of the heat build up

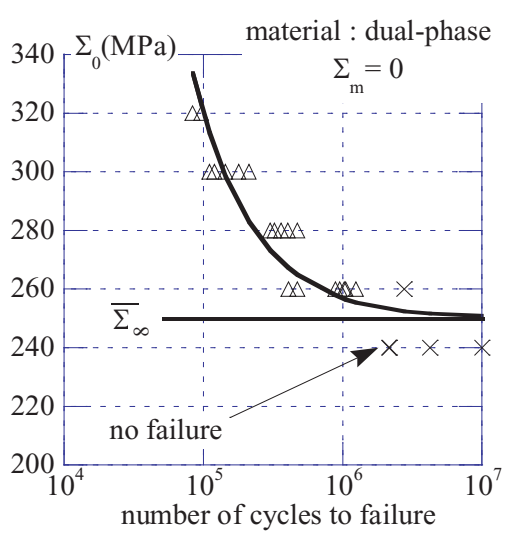

(d) Mean Wöhler curve

Figure 2: Empirical method to correlate heat build-up measurements to mean fatigue duration [2].

the current temperature of the sample during the test measured by a thermocouple or an infrared camera, $T_{1}$ and $T_{2}$ are the current temperatures of the lower and upper grips) is recorded and the steady-state temperature $\bar{\theta}$ is determined (figure 2(b) . Beyond a given limit it is observed that the steady-state temperature starts to increase significantly (figure 2(c)). This change is correlated with a state where the fatigue limit is exceeded and can be related to the apparition of microplasticity, i.e. plasticity at a microscopic scale, which occurrs in the material and governs the fatigue properties. A correlation between the mean fatigue is illustrated on figure 2(c) [3]. More recently, the development of a two-scale probabilistic model has shown that 
heat build-up tests permit to identify not only the mean fatigue limit but also the scatter of classical fatigue results. Indeed, by using the two-scale probabilistic model and an energetic criterion based on a constant critical dissipated energy, it is possible to predict the $\mathrm{S} / \mathrm{N}$ curves for any given probability of failure using a single specimen in less than half a day [2].

\subsection{Measurement protocol developed for rubber-like materials}

\subsubsection{Temperature measurement}

A heat build-up experiment can be defined as a succession of cyclic tests of increasing loading conditions during which the temperature of the specimen is measured. The number of cycles used for each loading condition is the number of cycles needed for the temperature to stabilize (for example, 2000 cycles at $2 \mathrm{~Hz}$ are sufficient). For the temperature measurement, we were facing two major technical problems: we have to set up an experiment that takes into account the large displacements of the specimen during a fatigue test and to properly define a heat build-up temperature. The responses to these problems were depending on the technological solution we retain for the temperature measurements. The use of thermocouples is possible but it presents some technological limitations (fixation on the specimen, response time of about $1 \mathrm{~s}$ for classical thermocouples) and the main point is that it only gives a local information. We have chosen to use an infrared camera, which gives access to a $2 \mathrm{D}$ measurement with a high acquisition rate (50 frames/seconds) and a very good precision (about $30 \mathrm{mK}$ ), even if it will only be a surface measurement. However, AE2 specimen are thinner enough to avoid a too high core/skin temperature ratio.

The infrared camera that has been used is a Flir Systems camera (reference Phoenix MWIR 9705) with a Stirling-cycle cooled Indium Antimonide (InSb) Focal Plane Array (FPA). The FPA is a $320 \times 256$ array of detectors digitized on 14 bits, sensitive in the $3 \mu \mathrm{m}-5 \mu \mathrm{m}$ spectral band. A preliminary calibration operation allows the conversion of the thermosignal (proportional to the thermal radiation) into a temperature in Celcius degree $\left({ }^{\circ} \mathrm{C}\right)$.

Figure 4 presents one example of temperature evolution of the central zone of the specimen during cyclic solicitation (20 cycles). We can note a mean rise of the temperature due to an intrinsic dissipation and an oscillation of the temperature during one cycle which is caused by the thermomechanical couplings (mainly thermoelastic effects). The amplitude of these coupling effects could attain one Celsius degree. We consequently had to make sure that the temperature is measured for a given position of the specimen to 


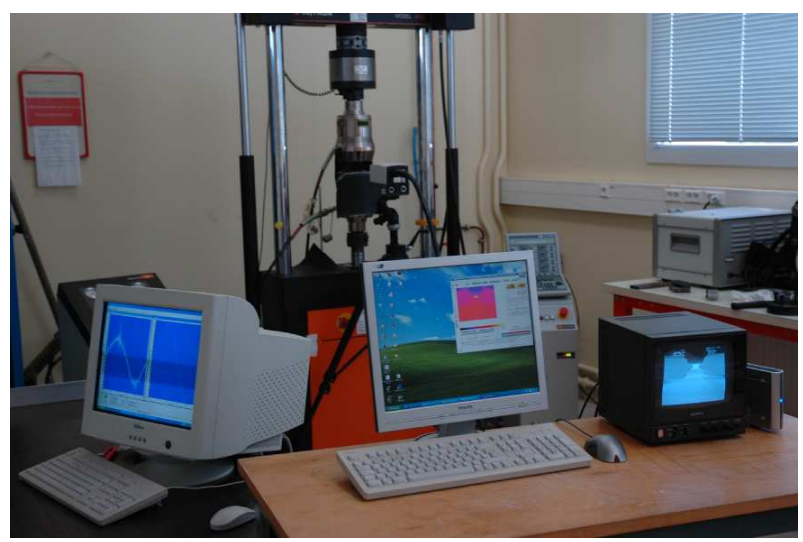

Figure 3: Experimental set-up.

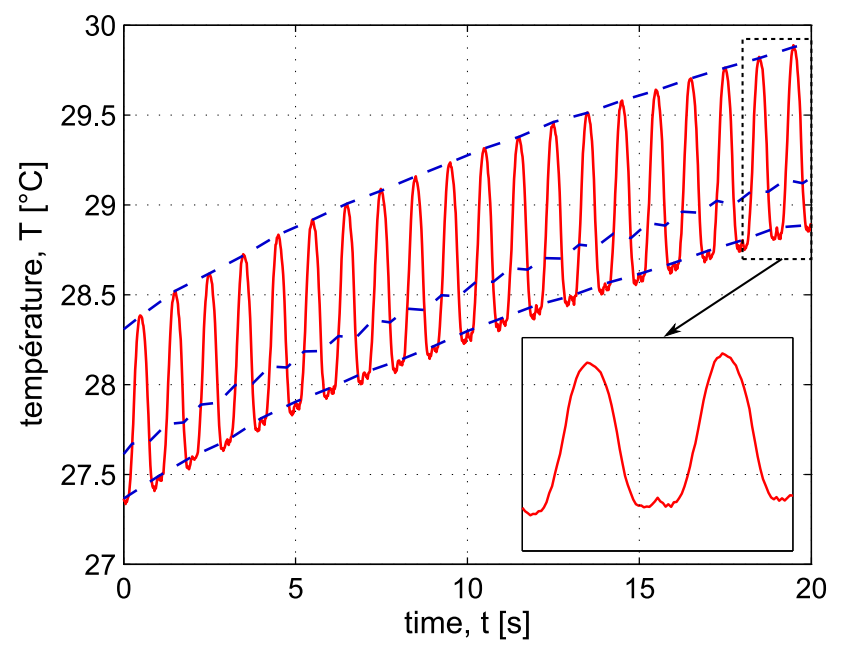

Figure 4: Evolution of the temperature of the central zone of the specimen during cyclic solicitation measured with an infrared camera $(R=0 @ 1 \mathrm{~Hz}$, maximum displacement: $10 \mathrm{~mm}$ ). The dotted lines correspond to the temperatures related to non-deformed, middeformed and deformed positions.

define accurately a heat build-up temperature. We used here sequences of movies (performed at an acquisition frequency of $50 \mathrm{~Hz}$ ), which allowed the evaluation of the temperature during one movie for any deformation of the sample (figure 5). For simplicity reasons and because all experiments are carried out at a loading ratio $R=0$, we have chosen to retain only temperatures associated to the non deformed (fig. 5(b) and maximum deformed position (fig. 5(c) of the specimen during one loading cycle. We should remark that 


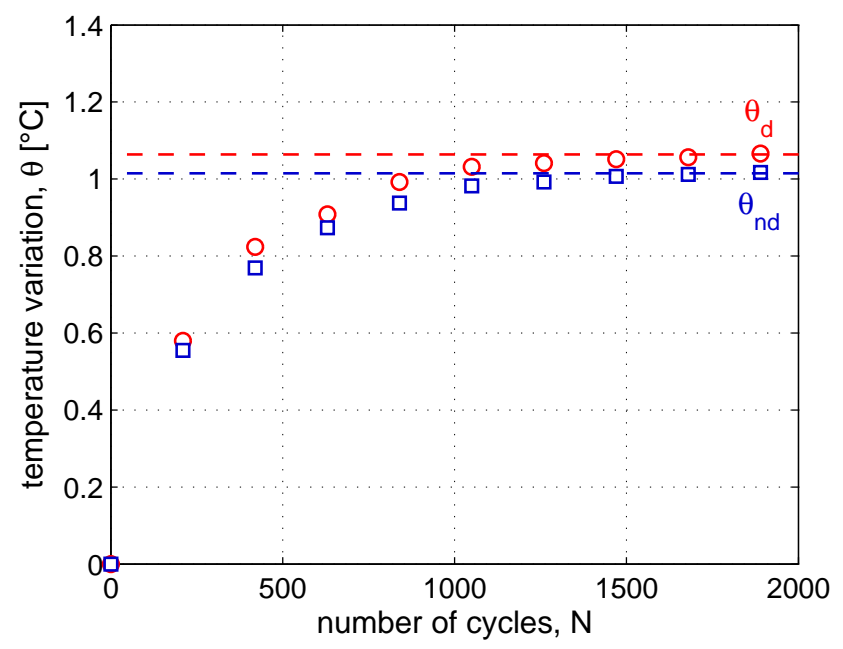

(a) Evolution of the temperature variation
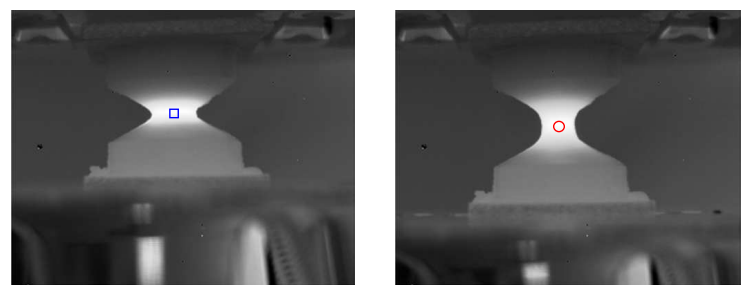

(b) non deformed posi- (c) maximum deformed tion position

Figure 5: Evolution of the temperature variation envelope for a value of displacement amplitude of $0.6 \mathrm{~mm}$.

the temperatures associated to these positions are not necessarily the minimum and maximum temperatures reached during one cycle because of the thermoelastic inversion phenomenon which can be clearly seen on figure 4 [4 6].

For each loading block, we get an envelope of temperature (figure 5(a)) and we evaluate the amplitude of the thermomechanical coupling effects (difference between the temperatures measured in the maximum deformed position and non-deformed position). The temperature variation is calculated from the current temperature $T$ and the initial temperature $T_{0}$ using the equation $\theta=T-T_{0}$. To take into account the global heating of the machine test, the temperature gradient of the specimen and the low thermal 
conductivity of rubber-like materials, a pause of $15 \mathrm{~min}$ is performed at the end of each loading block in order to ensure the temperature stabilization and homogeneity.

\subsubsection{Heat build-up curve construction}

Based on the successive loads carried out on an AE2 specimen, we can generate a heat build-up curve by associating a fatigue life predictor (for fatigue crack nucleation) with the steady-state temperatures. There is no universally used predictor in the literature. As the tests were displacement controlled, we chose the maximum principal strain $\varepsilon_{\max }$ which is one of the most commonly used [7 9] and which has been proven to be relevant for the AE2 specimen [9]. A heat build-up curve is therefore plotted from the steady-state temperatures as a function of $\varepsilon_{\max }$ as illustrated on figure 6 .

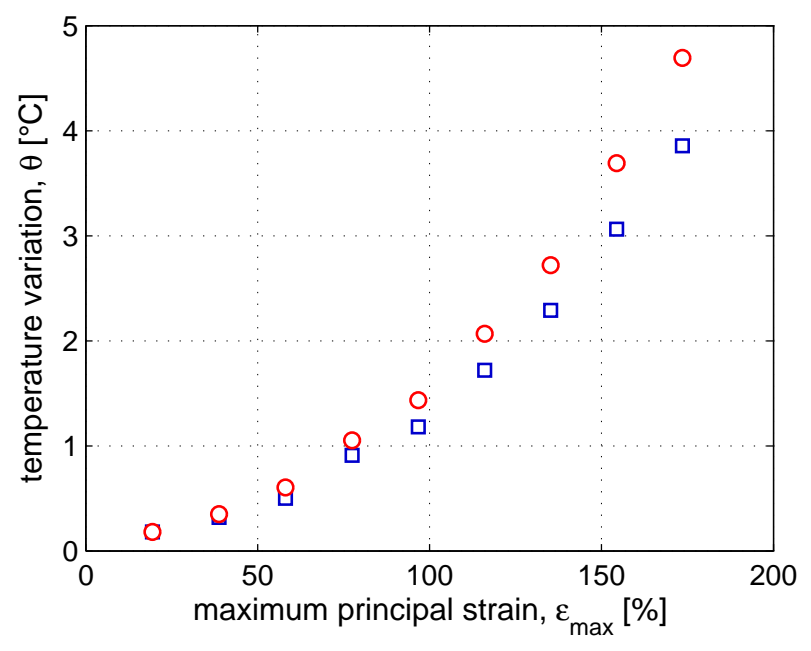

Figure 6: Heat build-up curve example.

\subsection{Mechanical testing}

All heat build-up tests were performed on a servo-hydraulic test machine (Instron 1342) at a frequency of $2 \mathrm{~Hz}$ and were dispacement controlled. The fatigue tests were performed at Trelleborg-Modyn laboratory on machines that have already been described elsewhere [10]. The experimental determination of the initiation Wöhler curve was achieved using a end-of-life criterion based on the variation of the effective stiffness proposed by [10]. It has been 
shown that the criterion is equivalent to the apparition of crack of $2 \mathrm{~mm}$ on the surface of the specimen [9].

\subsection{Tan $\delta$ measurements}

Tan $\delta$ is an indicator of the viscous potential of a material. Its value is not an intrinsic characteristic of a material but depends on the loading conditions. Consequently, it is very important to mention the test conditions related to the value of $\tan \delta$ that are used. All measurements are performed on compression specimens (diameter: $29 \mathrm{~mm}$ and height: $12 \mathrm{~mm}$ ). A static compression strain of $10 \%$ is applied and the dynamic load condition is a strain amplitude of $0.5 \%$ at $2 \mathrm{~Hz}$. The $\tan \delta$ value is then computed from the stress-strain response.

\section{Results and discussion on the thermal measurements}

\subsection{First observations}

In this paper, up to fifteen industrial materials have been tested. It was therefore mandatory to evaluate if the tests were repeatable in order to ensure the reliability of the differences between the different materials. This requirement was checked for several materials tested with small variations of the ambient temperature and for different loading histories (different numbers of loading blocks, with different increasing amplitudes). Figure 7 illustrates well that the heat build-up tests gave the same curves for these different testing conditions.

\subsection{A direct link with fatigue?}

At this stage of the study, we were evaluating if a quick empirical protocol similar to the one used for metallic materials could be used. In order to take into account several kinds of material and different thermo-mechanical properties, a wide range of materials was investigated (more than fifteen industrial materials were tested). We followed here a threefold approach:

- identify an empirical protocol giving the maximum strain leading to a damage initiation after $10^{6}$ cycles for a given material;

- apply this protocol on several materials in order to test its validity;

- investigate its sensitivity to the material viscosity. 


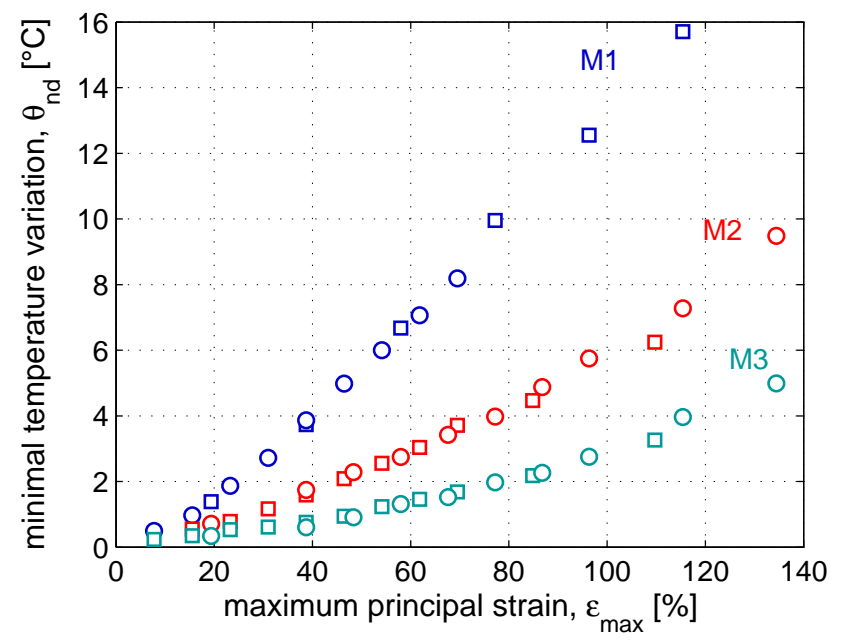

Figure 7: Comparison, for threee materials (referenced as M1, M2 and M3) of the heat build-up curves of two different specimens submitted to different loading conditions. For legibility reasons, only the temperatures associated to the non-deformed positions of the specimens are plotted.

Figure 8 presents the temperature measurements obtained for material G (cf. table 1) and a proposal of empirical analysis, similar to the one proposed for metallic materials. The material $\mathrm{G}$ was chosen because it presents a $\tan \delta$

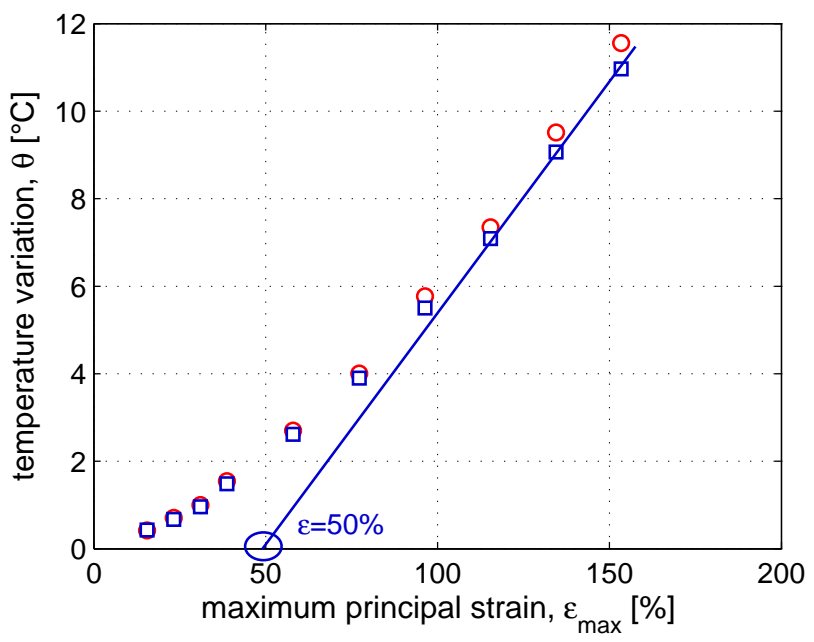

Figure 8: Empirical processing performed on material G. 
of average value. As the fatigue mechanisms are dissipative, we used only the rise of temperature corresponding to the dissipative sources (square symbols on figure 6), excluding the thermo-elastic contribution. One can see that the evolution of the heat build-up curve is much smoother than for metallic materials. This was expected as no clear fatigue limit is to be seen on the Wöhler curves obtained for elastomers. Moreover, rubbers exhibit many dissipative sources that will be discussed in paragraph 2.3, which makes the evaluation of the fatigue mechanism more difficult to point out than for metallics. Nevertheless, we were leading an exploratory study and we therefore adjusted an empirical analysis in order to meet the $10^{6}$ cycles strain identified on a Wöhler curve (cf. figure 8 and column 4 of table 10). This

\begin{tabular}{cccccc}
\hline reference & $\tan \delta$ & $\mathrm{Ni}(100 \%)$ & $\varepsilon_{106}$ cycles & $\varepsilon(A E)$ & error \\
\hline $\mathrm{A}$ & $<1$ & $10^{6}$ & $100 \%$ & $100 \%$ & $0 \%$ \\
$\mathrm{~B}$ & 2.3 & 237000 & $60 \%$ & $69 \%$ & $15 \%$ \\
$\mathrm{C}$ & 3 & 170000 & $55 \%$ & $62.5 \%$ & $13.6 \%$ \\
$\mathrm{D}$ & 4 & 123000 & $44 \%$ & $55 \%$ & $25 \%$ \\
$\mathrm{E}$ & 6.6 & 83600 & $32 \%$ & $30 \%$ & $6.2 \%$ \\
$\mathrm{~F}$ & 12.9 & 52000 & $30 \%$ & $30 \%$ & $6.6 \%$ \\
$\mathrm{G}$ & 12.9 & 16000 & $55 \%$ & $50 \%$ & $9 \%$ \\
$\mathrm{H}$ & 23.3 & 8000 & $20 \%$ & $30 \%$ & $33.3 \%$ \\
\hline
\end{tabular}

Table 1: Comparison between the $10^{6}$ cycles strain identified on a Wöhler curve and the estimated $10^{6}$ cycles strain using the heat build-up test.

protocol was then used to analyse the heat build-up curves obtained from seven other materials, presented on figure 9. The results and the materials data are afforded in table 1. It can be observed from the good agreement with the values provided by the Wöhler curves that the proposed graphical analysis is giving surprisingly good results. What can also be noticed is that the materials used present the same ranking from a fatigue point of view or from a viscous point of view (evaluated from their $\tan \delta$ ). As this fact was observed for the other tested materials (not presented here), a not yet resolved question is: what is really measured during a heat build-up test? To give some clues on that question, other tests have been performed on selected materials showing either the same $\tan \delta$ but different fatigue resistance, or the same fatigue resistance but with different $\tan \delta$. This is what is presented in the next paragraph. 


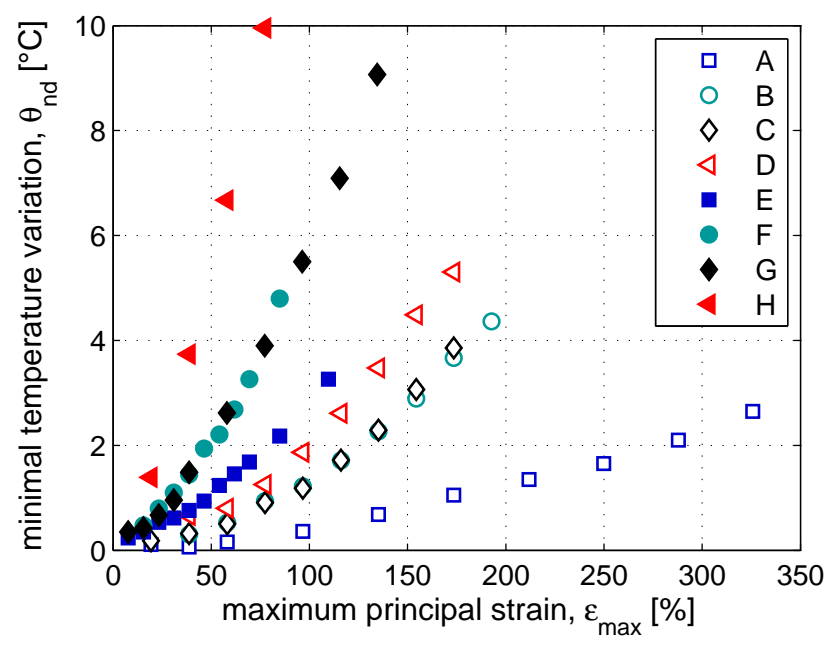

Figure 9: Heat build-up curves for materials A to $\mathrm{H}$.

\subsection{Sensitivity of the empirical protocol to the viscous dissipation}

Rubber-like materials are known to be hysteretic materials at a macroscopic scale, which means that some energy is dissipated and/or stored during one loading cycle. This hysteresis is not well explained and can be justified by several explanations:

(a) Mullins effect [11]

(b) crystallization under strain [12]

(c) viscosity 13

(d) plasticity 14

(e) damage [15]

The contribution of each of these sources is not clearly defined yet but it is generally assumed that viscosity is one of the most important [13] and is a first order parameter. To check this assumption, we performed heat build-up experiments on specifically selected materials that have equivalent viscous dissipation potential (evaluated from the $\tan \delta$ value) and different fatigue lifetimes and materials that have different viscous dissipation potentials (evaluated from the $\tan \delta$ value) and equivalent fatigue lifetimes. The figure 10 shows the experimental curves obtained on materials having different $\tan \delta$ but the same fatigue lifetime. It seems that the empirical protocol is working quite well. Considering now materials that have the same $\tan \delta$ but different fatigue lifetimes (see figure 11), the curves obtained are very similar and can 


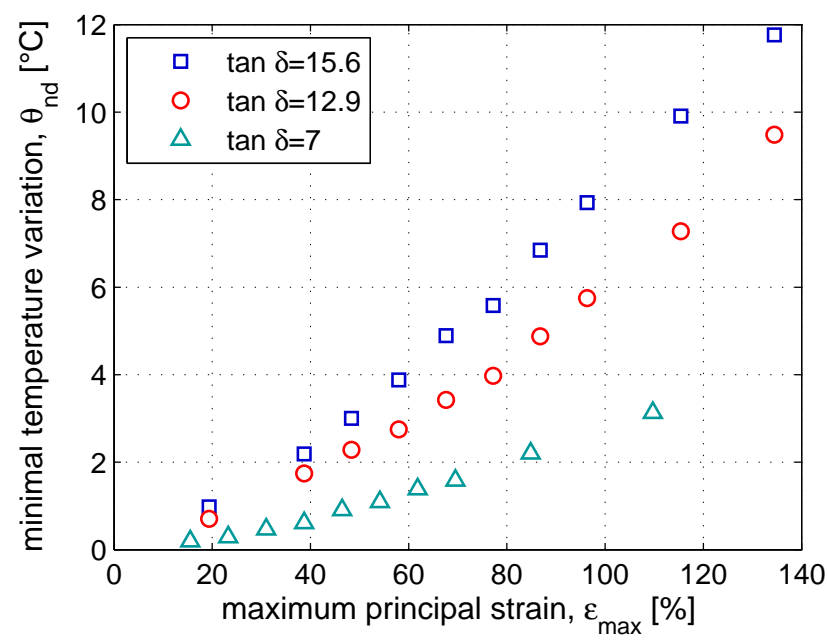

Figure 10: Heat build-up curves for materials having the same fatigue lifetime but different $\tan \delta$.

not lead to isolate the fatigue lifetime. It can therefore be concluded that

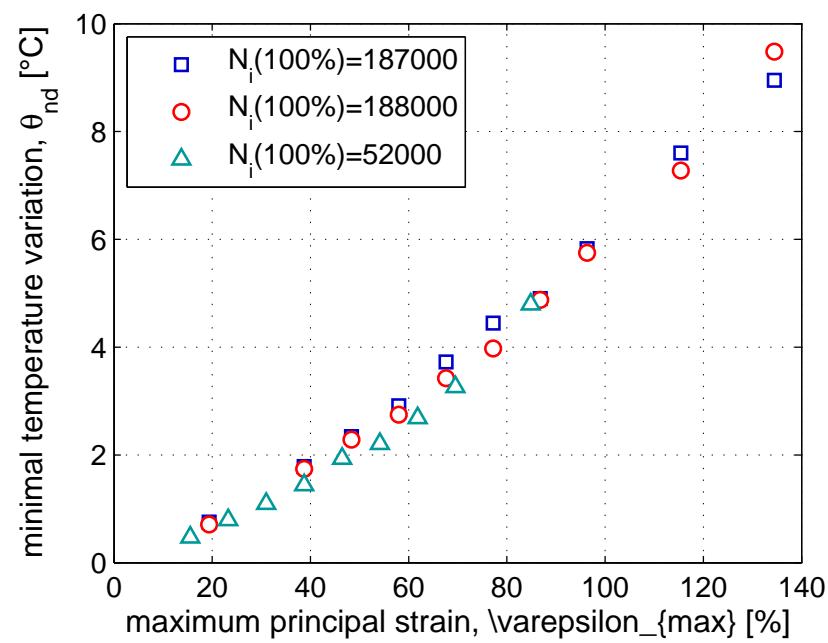

Figure 11: Heat build-up curves for materials having the same $\tan \delta$ but different fatigue lifetime.

even if the rough estimation analysis presented above has proven to be quite effective for several materials, a clear separation between viscous dissipation and a dissipation related to the fatigue resistance is far less easy to identify 
for rubber-like materials than for metallic materials. Moreover, this protocol is giving only one point of the Wöhler curve, which is clearly not enough as the slopes of these curves may be quite different depending on the nature of the tested rubber [16]. Nevertheless, the heat build-up measurements are useful to identify the parameters of constitutive models including some dissipation sources (viscosity, plasticity, damage) because these tests provide both mechanical and thermal data. Moreover, a stabilization of the temperature increase was always observed (at the limited frequency imposed) and this stabilization means that the dissipation source is nearly constant: an equilibrium between what is lost by conduction and convection and induced by cyclic loadings is reached. It is also clear that the global dissipated energy could not be directly related to the fatigue damage and that the description of the damage at lower scales is mandatory. In paragraph 3 we present how the temperature measurements can be useful if some more information on the fatigue damage evolution at a microscopic scale is provided.

\section{Evolution of fatigue damage using X-ray microtomography}

\subsection{Principle of the critical energy criterion and scope of the investigation}

Basically, fatigue design of rubber componentsrequires two steps: first choose a mechanical parameter: strain [7, 10], stress [17 19], strain energy [18, 20] . . . (see paragraph 1.3.2), and second perform several fatigue tests on samples of different geometries, under different sollicitations, in order to generate Wöhler curves, plotting the value of the chosen parameter with respect to the number of cycles needed to break the sample or to initiate a crack. A power law is then usually identified, relating the parameter to the number of cycles and is to be applied to design industrial components. It is important to note that the fatigue parameter can be different from the initiation criterion. This initiation criterion is usually a damage parameter, chosen for its ability to be summed in order to analyze variable fatigue solicitations. Depending on the materials, this parameter could be the dissipated energy [21 23], the cumulated plastic strain [24] or a scalar damage parameter associated with an evolution law [25]. Here, we will keep the maximal strain chosen formerly as the fatigue parameter and we will use an initiation criterion based on the cumulated dissipated energy. The principle of the approach is therefore very classic and simple: whatever the fatigue parameter may be, the cumulated dissipative energy needed to initiate a crack will be a constant, called here CDE (Critical Dissipated Energy). This energy based approach is close to 
the one proposed by [26] based on a Cracking Energy Density (CED) which is cumulated along the cycles and aims at being representative of the crack opening. It can also be related to the approach proposed by [15].

In elastomers, the energy dissipated evolves during the fatigue tests but reaches a stabilized value after the number of cycles needed to stabilize the rise of temperature induced by the heat build-up. Once this stabilization step is achieved, the dissipated energy measured along the fatigue test is almost a constant, for a given global displacement. This is confirmed by the stabilization of the heat build-up along a fatigue test, showing that the dissipation sources are constant (see figure 5(a)). It is therefore possible to write that:

$$
\mathrm{CDE}=N \cdot E_{\text {fatigue,diss/cycle }}
$$

with CDE the Critical Dissipated Energy considered as an intrinsic constant, $N$ the number of fatigue cycles needed to reach the initiation (according to a given experimental criterion) and $E_{\text {fatigue,diss/cycle }}$ the energy dissipated per cycle by the fatigue mechanisms, which is a function of the maximum strain.

As illustrated in paragraph 2, the dissipated energy is not easy to relate to the fatigue durability of elastomers as it does not depend only on the fatigue mechanisms. Several materials exhibiting the same dissipated energy per cycle will not necessarily have the same fatigue lifetime. It is consequently difficult to propose an experimental evaluation of the value of $E_{\text {fatigue,diss/cycle }}$ introduced in equation 1 since both a global evaluation of the dissipated energy and reliable micro-structural data on the fatigue mechanisms are required. The proposed approach is therefore:

- to use micro-structural measurements of the fatigue defect population, as it is well stated in the literature that the fatigue mechanisms for both initiation and propagation at a micro-scale are strongly related to the defect population [16, 27 32];

- to use the heat build-up measurements as an evaluation of the dissipated energy during cyclic and fatigue tests.

We focus here on a polychloroprene rubber, which was studied during the heat build-up campaign and which fatigue damage evolution was followed by X-ray computed tomography [1]. The samples used for the fatigue tests, the heat build-up tests and the X-ray tomography measurements were obtained from the same batch of material. The complex shape of the specimen (see 
figure 11) ensured that the initiation zone is well controlled and is located in the thinner section. Temperature measurements were also achieved in that zone, and the defects populations presented in the following are only the ones observed in slices of $1 \mathrm{~mm}$ thick, located the middles of the samples (see figure 12). It is therefore possible to link the observations made for the fatigue

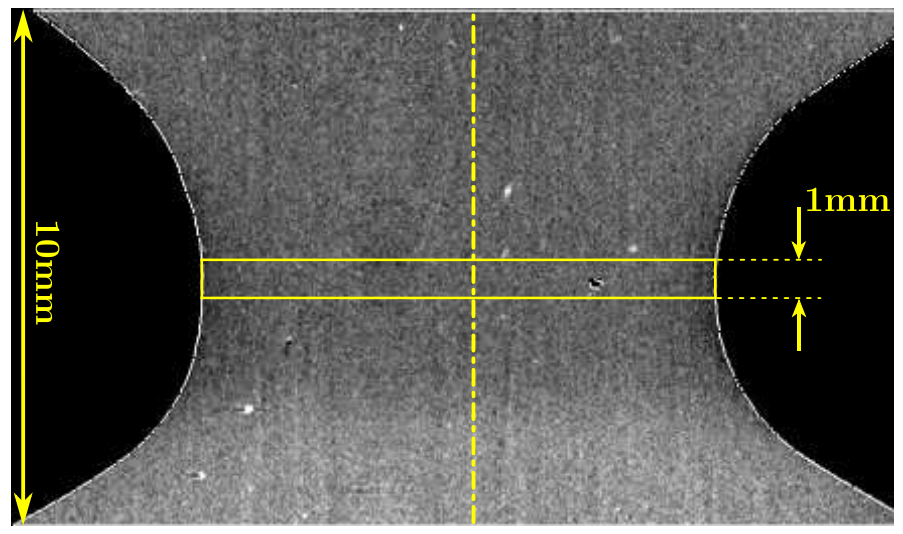

Figure 12: Studied zone for the temperature and tomography measurements.

initiation lifetime, the temperature rise and the defect population. In the following, the defect population dependency on the maximum strain and on the number of cycles is detailed (paragraph 3.2), then the main assumptions

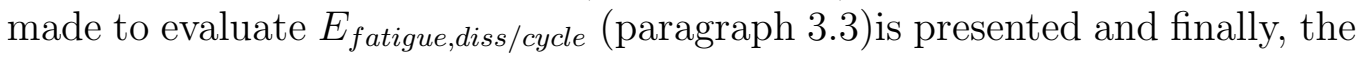
identification of the Critical Energy Density is achieved in paragraph 3.4. Finally a validation of our method is proposed by comparing the initiation curve built from our approach to the experimental fatigue results.

\subsection{Volumic defect density}

X-ray computed micro-tomography was used to investigate the fatigue damage occurring in a polychloroprene rubber [1]. This non destructive technique allowed us to study the evolution of the defect population along the fatigue cycles and its dependency on the maximum local strain. This study revealed that the volumic defect density depends both on the number of cycles and on the maximum local strain. It also showed that the defect density was evolving quickly during the first step of the fatigue test (less than 10\%) and was then evolving very slowly. The value obtained after a few thousands cycle is therefore representative of the initiation value. Moreover, the evolution of the defect volumic density with respect to the maximum 
strain was measured. This curve is plotted on figure 13 and was obtained from several samples and for several fatigue test durations. We also checked

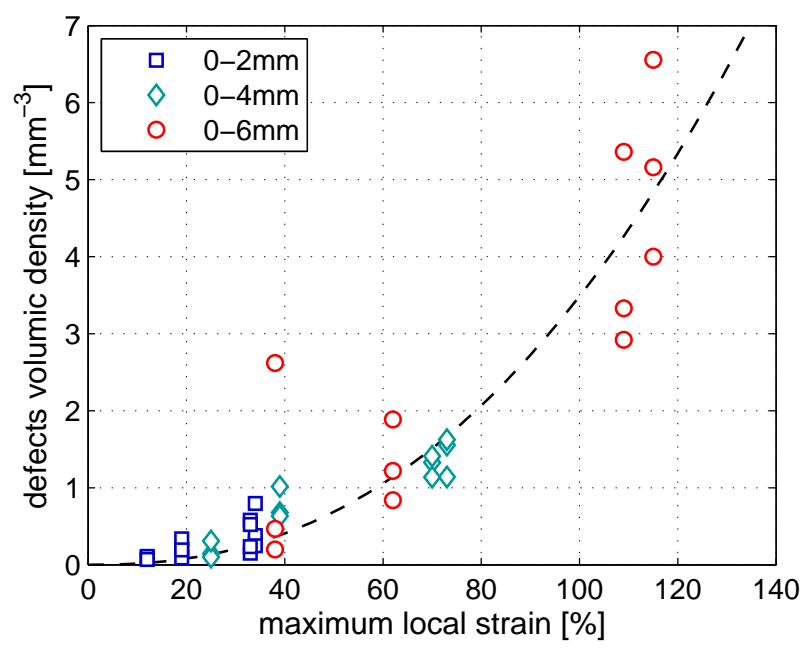

Figure 13: Evolution of the defect population density with respect to the maximum principal strain. The legend values correspond to the macroscopic displacement applied to the specimen.

that the average radius of the defects was not evolving much along the fatigue test and was not dependent on the maximum strain. The defect density is used here to be more precise but as the studied volume is the same for all the samples, it can also be seen as the number of defects. We therefore know the dependency of the defects density on the maximum strain but we still do not know how the material dissipates energy.

\subsection{Evaluation of the energy dissipated by a defect population}

Once the defect population and its evolution along the maximum local strain are described, a first way to evaluate the $E_{\text {fatigue,diss/cycle }}$ would be to write that :

$$
E_{\text {fatigue,diss/cycle }}=\sum_{\text {defects }} E_{\text {diss/cycle }}^{d}
$$

with $E_{\text {fatigue,diss/cycle }}^{d}$ being the dissipated energy by each defect.

The next step combines Finite Element simulations [28, 33] and homogeneization techniques [34]. This is maybe the closest approach from the microscopic mechanisms but it is still meeting very big difficulties. The first 
problem comes from the assumed equivalence between a given population of defects exhibiting different sizes and shapes and a homogeneous population of an equivalent defect. Then, the evaluation of the energy dissipated by a given defect meets the complexity of elastomeric materials (shape of inclusions, matrix visco-hyper-elasticity, low compressible behaviour, difficulty to identify the interphase behaviour, necessity to take the inclusion interactions into account, ...). At last, the dissipative sources related to a defect in an elastomeric matrix are still not well identified (growth of the cavity, matrix inelasticity, friction at the interface ...) and are therefore difficult to evaluate. Here, we have chosen a much more phenomenological approach: we chose to relate the total dissipated energy (evaluated from the stabilized rise of temperature) to the energy dissipated by the defect population.

The first hypothesis assumes that the ratio between the total dissipated energy and the one related to the fatigue mechanisms (whatever the kind of dissipation it leads to) is linearly dependant on the defect density. It would then come:

$$
E_{\text {fatigue }, \text { diss } / \text { cycle }}=A \cdot \mathrm{DVD} \cdot V \cdot E_{\text {diss } / \text { cycle }}
$$

With $A$ being a constant, DVD the Defects Volumic Density in the central zone of the sample, $V$ the considered volume and $E_{\text {diss } / c y c l e}$ the total dissipated energy during one fatigue cycle in that volume. The second hypothesis is that the total dissipated energy is evaluated from the temperature rise only. This strong assumption is supported by the very low thermal conductivity of the material and because we consider here only the rise of temperature linked to the dissipation, with no thermoelastic couplings (see paragraph 1.3.1). This point will of course be further investigated but this assumption is taken as a first rough evaluation. We could consequently write that:

$$
E_{\text {diss } / \text { cycle }}=B \cdot \Delta T
$$

With $B$ a constant parameter and $T$ the stabilized rise of temperature in the central zone of the sample, which is dependant on the maximum imposed deformation. This global evaluation is also required because:

- the rise of temperature, measured at the skin, is an average value of the dissipation sources that are located in the volume underneath;

- the defect volumic density is also defined as an average value for a given volume. 
From equations 1, 3 and 4, it would then come:

$$
\mathrm{CDE}=N \cdot A \cdot \mathrm{DVD} \cdot V \cdot B \cdot \Delta T
$$

Which can also be written as:

$$
N \cdot \mathrm{DVD} \cdot \Delta T=c^{\text {ste }}
$$

for a given volume.

\subsection{Experimental validation of our approach}

In the following we will use the figure 13, 14and15in order to evaluate the numerical values of the terms in equation 6. The initiation lifetime is taken from the Wöhler curve, the defect volumic density is obtained from the curve fitted on the X-ray tomography measurements and the rise of temperature is obtained from the heat-build up curve. The first step is to identify the value of the constant of equation 6 , that is given in table 2 . In order to

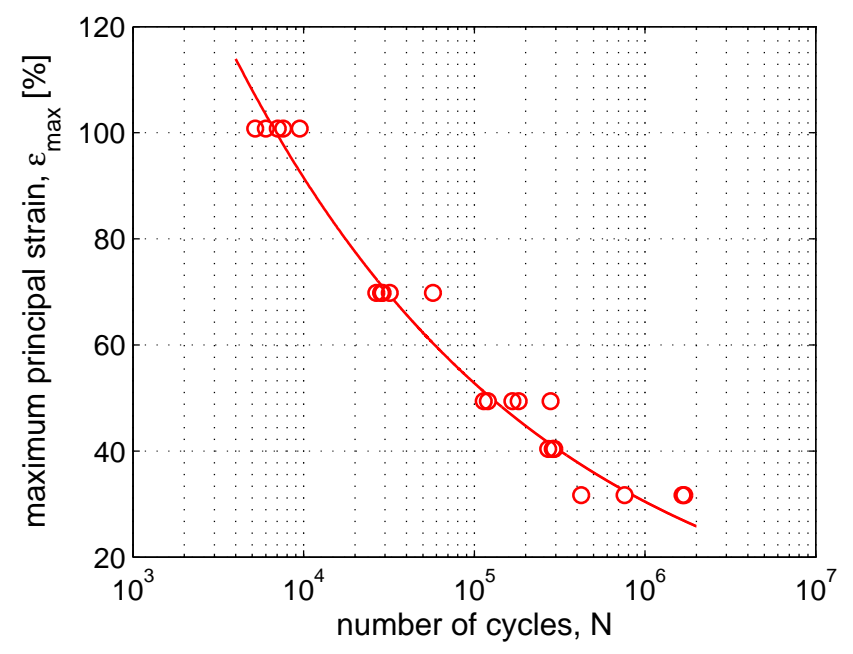

Figure 14: Wöhler curve of the studied polychloroprene rubber.

validate the approach, we then evaluate the product of equation 6 for several local maximum strains. It can be observed from table 2 that the values obtained are very similar. The second step of validation was to build an initiation curve from equation 6, using one of the constant evaluated (for example 440000, obtained for a $2 \mathrm{~mm}$ displacement) and power laws fitted 


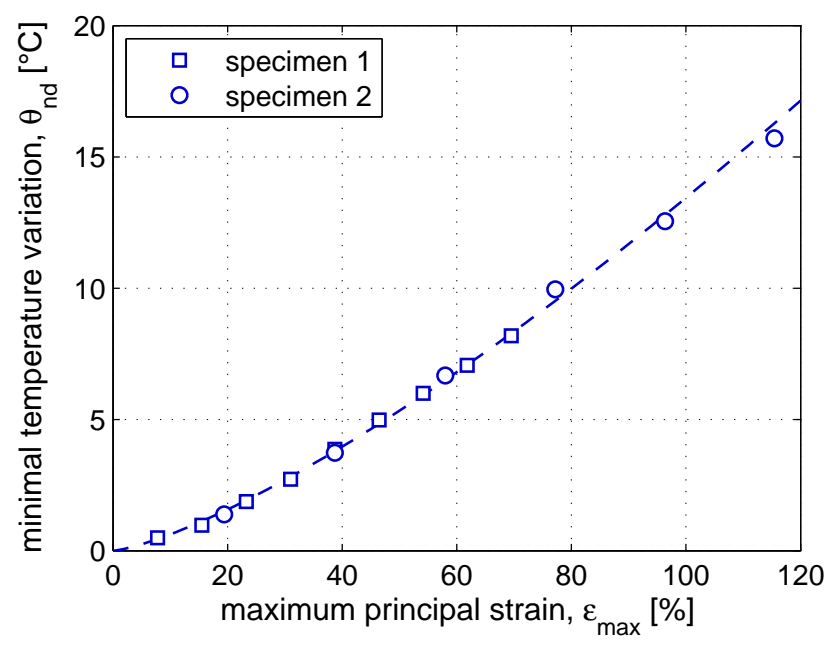

Figure 15: Heat build-up curve of the studied polychloroprene rubber.

\begin{tabular}{ccccc}
\hline$\varepsilon_{\max }$ & $\mathrm{Ni}$ & $\mathrm{DVD}$ & $\bar{\theta}_{n d}$ & evaluated energy based constant \\
\hline $30 \%$ & $10^{6}$ cycles & $0.2 \mathrm{~mm}^{-3}$ & $2.2^{\circ} \mathrm{C}$ & 440000 \\
$70 \%$ & $3.5 \cdot 10^{4}$ cycles & $1.5 \mathrm{~mm}^{-3}$ & $8.2^{\circ} \mathrm{C}$ & 430500 \\
$110 \%$ & $6.5 \cdot 10^{3}$ cycles & $4.5 \mathrm{~mm}^{-3}$ & $15.2^{\circ} \mathrm{C}$ & 444600 \\
\hline
\end{tabular}

Table 2: Evaluation of the Critical Dissipated Energy

on the curves of figure 13 (defects density) and 14 (stabilized temperature rise). The curve obtained is plotted on figure 16 and exhibits a very good correlation with the other experimental points. As the value obtained from equation 6 was varying a little from a condition to another, we also plot the same curve, obtained with a variation of $15 \%$ from the value of the constant used to build the curve. It illustrates that the variations observed in table 2 lead to very few differences of the reconstructed initiation lifetime curve.

Despite the very strong hypotheses used here and that need to be further investigated, this approach leads to convincing results and seems therefore very promising because it could shorten very much the determination of the fatigue properties of an elastomeric material. With about 6 samples in order to propose a reliable fit of the evolution of the defects population along the maximum strain (5 submitted to an interrupted heat build-up protocol and then analysed by X-ray tomography, 1 that is pushed untill the initiation in order to have a value for the energy constant) and within 3 days (one 


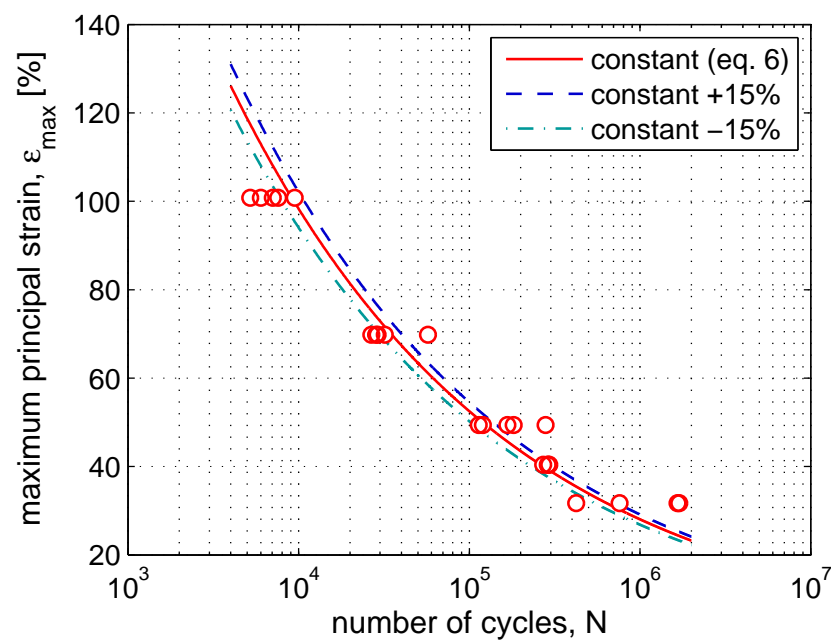

Figure 16: Prediction of the Wöhler curve using the proposed approach.

day for mechanical testing and 2 for X-ray measurements and analysis) it could be possible to evaluate the full initiation lifetime curve. The dispersion observed for fatigue tests might also be deduced from the dispersion on the defect density, as the rise of temperature is very repeatable. Nevertheless, the proposed approach is of course to be validated on several materials, which is currently undertaken.

\section{Conclusions}

In this study we focused on the development of a heat build-up test, which relates the maximum principal strain to the temperature rise. A very first step was to propose an accurate measurement protocol suitable for large displacements and that could discriminate the temperature rise induced by cumulative dissipation from the thermo-elastic contribution. More than fifteen industrial materials were tested in order to experiment the ability of that kind of test to be representative of the fatigue resistance of elastomers. These tests provided promising results but a quick analysis is still made delicate by the highly viscous nature of these materials. In order to propose a physically based evaluation of the dissipated energy related to the fatigue defects, we used here the results from another paper [1]. The defect volumic density was used to evaluate the ratio of the total dissipated energy, deduced from the heat build-up measurements, that could be related to the 
damage. An energy based fatigue criterion is then proposed and validated for a polychloroprene rubber. The correlation between the fatigue results obtained from a classical Wöhler curve and those from the proposed approach is excellent and clearly calls further developments and validations on other materials. This study also illustrated that the heat build-up test seems to be an appropriate tool to identify the parameters of constitutive models including dissipation, as both mechanical and thermal responses are analysed. It also proved how valuable micro-tomography measurements can be in order to understand what happens during the heat build-up tests and to feed the mechanical models with damage kinetics.

\begin{tabular}{|c|c|c|c|c|}
\hline reference & polymer & Fillers & Vulc. system & Appl \\
\hline A (fig. 9) & NR (100 phr) & 0 & Conv. & reast \\
\hline B (fig. 9) & NR (100 phr) & CB (22 phr) & Conv. & $\mathrm{P}$ \\
\hline C (fig. 9) & NR (100 phr) & CB (21 phr) & $\mathrm{S}-\mathrm{EV}$ & $\mathrm{P}$ \\
\hline D (fig. 9) & NR (100 phr) & CB (28 phr) & $\mathrm{EV}$ & $D$ \\
\hline E (fig. 9) & NR (100 phr) & CB (38 phr) & $\mathrm{EV}$ & $\mathrm{P}$ \\
\hline F (fig. 9) & NR (100 phr) & $\mathrm{CB}(55 \mathrm{phr})+\mathrm{Si}(12 \mathrm{phr})$ & EV-S-EV & \\
\hline G (fig. 9) & NR (100 phr) & $\mathrm{CB}(43 \mathrm{phr})$ & Conv. & $P$ \\
\hline H (fig. 9) & CR (100 phr) & & & Offshore \\
\hline $\tan \delta=15.6$ (fig. 10) & NR (100 phr) & CB (56 phr) & S-EV-Conv. & \\
\hline $\tan \delta=12.9$ (fig. 10) & NR (100 phr) & CB (50 phr) & S-EV-Conv. & \\
\hline $\tan \delta=7$ (fig. 10$)$ & NR (100 phr) & $\mathrm{CB}(25 \mathrm{phr})$ & S-EV-Conv. & \\
\hline$N_{i}=187000$ (fig. 11) & NR (100 phr) & CB (N339-55 phr) & S-EV-Conv. & \\
\hline$N_{i}=188000$ (fig. 11) & NR (100 phr) & Cb (N339-50 phr) & S-EV-Conv. & \\
\hline$N_{i}=52000($ fig. 11) & NR (100 phr) & CB (N650-55 phr) & S-EV-Conv. & \\
\hline
\end{tabular}

Table 3: Compounds specifications. Notations : NR (Natural Rubber), Cr (Polychloroprene Rubber), CB (Carbon Blacks), Conv. (Convecntional), S-EV (Semi Efficient), EV (Efficient), PTMS (Power Train Mounting System), PSS (Power Strut System)

\section{Acknowledgements}

The authors would like to thank the Britanny region for its financial support and all the actors of the FEMEM project. A special thank to P. Laguillaumie for his carefull reading. 


\section{References}

[1] V. Le Saux, Y. Marco, S. Calloch, P. Charrier, Evaluation of the fatigue defects population in an elastomer using X-ray computed microtomography, International Journal of Fatigue Submitted (July 2009).

[2] C. Doudard, S. Calloch, P. Cugy, A. Galtier, F. Hild, A probabilistic twoscale model for high-cycle fatigue life predictions, Fatigue \& Fracture of Engineering Materials \& Structures 28 (2005) 279-288.

[3] M. Luong, Infrared thermography of fatigue in metals, SPIE 1682 (1992) $222-233$.

[4] J. Joule, On some thermodynamics properties of solids, Philosophical Transactions of the Royal Society of London 149 (1859) 91-131.

[5] R. Anthony, R. Caston, E. Guth, Equations of state for natural and synthetic rubber-like materials. I. Unaccelerated natural soft rubber, The Journal of Physical Chemistry 46 (1942) 826-840.

[6] L. Treloar, The physics of rubber elasticity (third edition), Oxford (UK) : Oxford University Press, 1975.

[7] S. Cadwell, R. Merrill, C. Sloman, F. Yost, Dynamic fatigue life of rubber, Industrial and Engineering Chemistry 12 (1940) 19-23.

[8] B. Roberts, J. Benzies, The relationship between uniaxial and equibiaxial fatigue in gum and carbon black filled vulcanizates, Proceedings of Rubbercon'77 (1977) 2.1-2.13.

[9] E. Ostoja Kuczynski, Comportement en fatigue des élastomères : application aux structures antivibratoires pour l'automobile, Ph.D. thesis, Ecole Centrale de Nantes, Université de Nantes, 2005.

[10] E. Ostoja Kuczynski, P. Charrier, E. Verron, L. Gornet, G. Marckmann, Crack initiation in filled natural rubber: experimental database and macroscopic observations, in: Constitutive Model for Rubber III, London (UK), 3-10, 2003.

[11] J. Diani, B. Fayolle, P. Gilormini, A review on the Mullins effect, European Polymer Journal 45 (2009) 601-612. 
[12] J. Marchal, Cristallisation des caoutchoucs chargés et non chargés sous contraintes : effet sur les chaînes amorphes, Ph.D. thesis, Université de Paris XI Orsay, 2006.

[13] J. Bergström, M. Boyce, Constitutive modeling of the large strain timedependend behavior of elastomers, Journal of the Mechanics and Physics of Solids 46 (1998) 931-954.

[14] C. Miehe, J. Keck, Superimposed finite elastic-viscoelastic-plastoelastic stress response with damage in filled rubbery polymers. Experiments, modelling and algorithmic implementation, Journal of the Mechanics and Physics of Solids 48 (2000) 323.365.

[15] J. Grandcoin, Contribution à la modélisation du comportement dissipatif des élastomères chargés : d'une modélisation micro-physiquement motivée vers la caractérisation de la fatigue, Ph.D. thesis, Université d'Aix-Marseille II, 2008.

[16] W. Mars, A. Fatemi, A literature survey on fatigue analysis approaches for rubbers, International Journal of Fatigue 24 (2002) 949-961.

[17] C. Lu, Etude du comportement mécanique et des mécanismes d'endommagement des élastomères en fatigue et en fissuration par fatigue, Ph.D. thesis, CNAM, 1991.

[18] F. Abraham, T. Alshuth, S. Jerrams, The effect of minimum stress and stress amplitude on the fatigue life of non strain crystallising elastomers, Materials \& Design 26 (2005) 239-245.

[19] N. Saintier, G. Cailletaud, R. Piques, Multiaxial fatigue life prediction for a natural rubber, International Journal of Fatigue 28 (2006) 530-539.

[20] F. Lacroix, S. Méo, G. Berton, F. Chalon, A. Tougui, N. Ranganathan, A local criterion for fatigue crack initiation on chloroprene rubber : approach in dissipation, in: ECCMR IV, Stockholm (Sweden), 2005.

[21] F. Ellyin, K. Golos, Multiaxial fatigue damage criterion, Journal of Engineering Materials and Technology 110 (1988) 63-68.

[22] G. Fargione, A. Geraci, G. La Rosa, A. Risitano, Rapid determination of the fatigue curve by the thermographic method, International Journal of Fatigue 24 (2002) 11-19. 
[23] E. Charkaluk, A. Bigonnet, A. Constantinescu, K. Dang Van, Fatigue design of structures under thermomechanical loadings, Fatigue \& Fracture of Engineering Materials and Structures 25 (12) (2002) 1199-1206.

[24] I. Papadopoulos, Fatigue polycyclique des métaux: une nouvelle approche, Ph.D. thesis, Ecole Nationale des Ponts et Chaussées, 1987.

[25] J. Lemaitre, J. Chaboche, Aspect phénoménologique de la rupture par endommagement, Journal de Mécanique Appliquée 2 (3) (1978) 317365 .

[26] W. Mars, Multiaxial fatigue of rubber, Ph.D. thesis, University of Toledo, 2001.

[27] J. Busfield, A. Thomas, M. Ngah, Application of fracture mechanics for the fatigue life prediction of carbon black filled elastomers, in: Constitutive Model for Rubber, Rotterdam (The Netherlands), 249-256, 1999.

[28] N. Saintier, Fatigue multiaxiale dans un élastomère de type NR chargé : mécanismes d'endommagement et critère local d'amorçage de fissure, Ph.D. thesis, Ecole Nationale Supérieure des Mines de Paris, 2001.

[29] J. Le Cam, B. Huneau, E. Verron, L. Gornet, Mechanism of fatigue crack growth in carbon black filled rubber, Macromolecules 37 (2004) 5011-5017.

[30] J. Le Cam, E. Verron, B. Huneau, L. Gornet, Micro-mechanism of fatigue crack growth: comparison between carbon black filled NR SBR, in: Constitutive model for rubber IV, Stockholm (Sweden), 115-120, 2005.

[31] N. Saintier, G. Cailletaud, R. Piques, Crack initiation and propagation under multiaxial fatigue in a natural rubber, International Journal of Fatigue 28 (2006) 61-72.

[32] K. Le Gorgu Jago, Fatigue life of rubber components: 3D damage evolution from X-ray computed microtomography, in: Constitutive Model for Rubber V, Paris (France), 173-177, 2007. 
[33] Y. Fukahori, W. Seki, Stress analysis of elastomeric materials at large extensions using the finite elements methods. II: Stress and strain distribution around rigid spherical particles, Journal of Materials Science 28 (1993) 4471-4482.

[34] B. Omnès, S. Thuillier, P. Pilvin, G. Gillet, Non-linear mechanical behavior of carbon black reinfored elastomers: experiments and multiscale modelling, Plastics, Rubber and Composites 37 (2008) 251-258. 